\title{
The effect of nutrition and exercise on the level of glycogen in skeletal muscle of sheep
}

\author{
DW Pethick 1, JB Rowe 2 \\ 'School of Veterinary Studies, Murdoch University, 6150 Western Australia ; 2Dept Animal Science, \\ University of New England, Armidale, 2350 New South Wales, Australia
}

A high level of glycogen in skeletal muscle is important for the production of high quality meat by allowing the development of a low ultimate $\mathrm{pH}(\mathrm{pHu})$ (Warriss, 1990, Appl Anim Behav Sci, 28, 171- 186). Residual muscle glycogen is that remaining 48 hours post slaughter after the pHu has been achieved and high levels imply effective management systems for the maintenance of high glycogen levels. This study was designed to understand the effect of nutrition and exercise training on the level of glycogen in muscle. In this paper the effects on residual glycogen are reported.

Sixty four Merino wethers, 1 year old and starting body weight $38 \mathrm{~kg}$, were placed in individual pens and offered a ground and pelletised ration consisting of $20 \%$ straw, $26 \%$ lupin grain, $53 \%$ barely grain, $1 \%$ mineral/vitamin premix $(13.5 \%$ protein and $10.7 \mathrm{MJ}$ metabolisable energy (ME)/ $\mathrm{kg}$ as fed). The effects of nutrition and exercise were tested for 2 levels of exercise (sedentary versus exercised) and 4 levels of feed intake $(5.9,7.4,9.1$ and $13.2 \mathrm{MJ}$ of ME/day or $1,1.3$, 1.5 and $2.2 \times$ maintenance) with 8 sheep per treatment. Exercise consisted or $1 \mathrm{~h}$ at 7-8 $\mathrm{km} / \mathrm{h}$ (trot) 3 times per week and began in week 4 of the experiment and finished in week 9. The level of exercise was estimated as $70 \%$ $\mathrm{VO}_{2}$ max according to the equations of Jones et a/ (1992, J Appl Physiol, 73, 234-239). The sheep were slaughtered in week 10 at a local abattoir immediately after transport for 1 hour.
Samples of the $m$. semimembranosis (SM), $m$. semitendinosis (ST) and $m$. longissimus dorsi (LD) were taken $48 \mathrm{~h}$ post slaughter and used for the determination of glycogen content (Chan and Exton, 1976, Anal Biochem, 71, 96105). The results are shown below as mean \pm sem.

The levels of residual glycogen were similar for both the SM and LD but much lower in the ST as would be predicated from the nature of fibre types in each muscle. The SM and LD are described as type IIA muscle types while the ST is a type IIB (Braind et al, 1981, Eur J Appl Physiol, 46, 347-358). The ratio of Type I : IIA : IIB is $34: 45: 21$ and $21: 58: 22$ for the SM and ST respectively (Aalhus and Price, 1991, Meat Sci, 29, 43-56). The level of residual glycogen increased in a linear manner as the level of food intake increased from 1 times maintenance to ad libitum $(\mathrm{P}<0.01)$ with the response being greatest for the $S M$ and $L D$. Exercise training markedly increased residual glycogen level in skeletal muscle by 90,55 and $227 \%$ for the SM, LD and ST respectively $(P<0.001)$ with the magnitude greatest in the SM and LD.

In conclusion residual glycogen level in muscle is maximised by a combination of high nutrient intake and exercise training. The effects of exercise are probably related to the glycogen loading effects of exercise training (Tan et al, 1984, J Appl Physiol).
Feed Intake (x maintenance) ${ }^{a}$ Sedentary Exercised

1.0

1.3

1.5

2.2

$0.50 \pm 0.08 \quad 0.85 \pm 0.07$

$0.56 \pm 0.11 \quad 0.96 \pm 0.11$

\section{Residual muscle glycogen (\%)}

m. longissimus dorsi

m. semitendinosis

Sedentary Exercised Sedentary Exercised

$\begin{array}{ll}0.31 \pm 0.07 & 0.62 \pm 0.09\end{array}$

$0.28 \pm 0.04$

$0.52 \pm 0.11$

$10.00 \pm 0.00$
$0.08 \pm 0.03$

$0.35 \pm 0.09 \quad 0.73 \pm 0.08$

$0.36 \pm 0.07$

$0.60 \pm 0.06$

$0.08 \pm 0.04$

$0.10 \pm 0.05$

$0.51 \pm 0.09 \quad 0.62 \pm 0.06$

$0.07 \pm 0.04$

$0.28 \pm 0.06$

$0.55 \pm 0.09 \quad 0.81 \pm 0.11$

$0.18 \pm 0.06 \quad 0.29 \pm 0.05$

a maintenance was defined as the intake of $5.89 \mathrm{MJ}$ of ME/day for a $40 \mathrm{~kg}$ sheep. 